\title{
Parameterized Cellular Automata in Image Segmentation
}

\begin{abstract}
An original update rule for multi-state cellular automata is investigated in the context of grayscale image segmentation in this paper. The update rule is parameterized and takes into account the features of neighbouring cells compared to the features of the current cell. We apply the resulting cellular automata for segmenting several real-world images. In this process we study afterwards the influence of the rule parameters and cellular automata neighbourhood scheme by means of different evaluation measures.
\end{abstract}

\section{INTRODUCTION}

There is a huge amount of data that needs to be analyzed with the proper tools in order to generate knowledge. Image processing techniques of increasing complexity have therefore been emerging. Computer-aided image analysis, in particular, is used for diagnosis (e.g. breast cancer detection) or for reconstruction. From a computational point of view, these tasks are considered unsupervised classification problems (from a Machine Learning perspective) and image segmentation problems (from a Computer Vision perspective).

This paper represents a study of the way Cellular Automata can be used for image segmentation in general, and medical image segmentation in particular. Cellular Automata (CA) are decentralized structures of simple and locally interacting elements that evolve following a set of rules, emerging to a global behaviour. In this paper we illustrate ways in which Cellular Automata (CA) can be used for the segmentation of simple greyscale images.

The most popular neighbourhoods used in twodimensional CA are von Neumann and Moore. A von Neumann neighbourhood is the set of all cells that are orthogonally-adjacent to the core cell (the core cell itself may or may not be considered part of the neighbourhood, depending on the context). A Moore neighbourhood is the set of all cells which surround the core cell.

Image segmentation techniques are still far from being able to identify relevant features in medical images, such as tumours or even well-defined organs. It is in this feature identification process that we propose to make use of CA where, through appropriate choice of evolution rules and topologies, we can identify pixels which belong together. There are only few attempts in the literature at using binary CA for image segmentation [1], [2], [3], [4] however these confirm the scientific potential of the proposition. To the best of our knowledge, multi-state CA have not been used until now for image segmentation.

Segmentation algorithms found in the literature can be classified according various criteria: complete $v s$. partial segmentation [5]; global-knowledge-based vs. edge-based (identify pixels belonging to frontiers, followed by procedures to link them) or region-based (assign pixels to individual regions) [6]; fully automated methods (each algorithm has to use the same set of parameters for all test images) $v s$. interactive methods (also known as semi-supervised or semiautomated segmentation) [7].

Various tasks pertaining to binary images have been solved using CA [8]-[11], such as edge detection, noise filtering, thinning and finding the convex hulls of all regions. In [12][14] a thresholded local update rule is proposed for edge detection in greyscale images.

In [8] a threshold decomposition method is used in order to perform image processing tasks in greyscale images. Another greyscale image processing model is investigated in [15] based on a three-state CA. Examples of multi-state CA are: Sierpinski cellular automaton [16], a sandpile cellular automaton (or the Abelian sandpile model) [17], a GH automaton [18].

This paper analyses a recently proposed update rule to be used by CA. The rule is parameterized and takes into account features of neighbouring cells compared to the features of the current cell. Different values of the involved parameters along with different neighbourhood schemes are evaluated by the means of different performance evaluation measures and studied in this paper.

The paper is organised as follows. Section II formalizes the problem of image segmentation. Proposed rule is described in Section III. The description of used datasets and the results are discussed in Section IV. In Section V we conclude with a discussion around the validation results and future research directions.

\section{SCIENTIFIC PROBLEM}

The complete segmentation of an image $I$ can be defined as the partitioning of $I$ in $L$ subregions, $R^{1}, R^{2}, \ldots, R^{L}$, such that:

- $\bigcup_{l=1}^{n L} R^{l}=I$

- $\forall \widetilde{x} \in R^{l}, S(\widetilde{x})=l, \forall l \in\{1,2, \ldots, L\}$

- $\quad R^{l}$ is a connected set, $\forall l \in\{1,2, \ldots, L\}$

- $\quad R^{l_{1}} \cap R^{l_{2}}=\phi, \forall l_{1}, l_{2}, l_{1} \neq l_{2}$

- $Q\left(R^{l}\right)=$ True,$l \in\{1,2, \ldots, L\}$

- $Q\left(R^{l_{1}} \cup R^{l_{2}}\right)=$ False for each pair of adjacent regions $R^{l_{1}}, R^{l_{2}}$.

where $Q$ is a logical predicate defined on the points of the considered region $(Q$ is used for characterizing the objects of the image). 
The segmentation problem can be considered solved when a label is associated to every pixel. The number of labels may or may not correspond to the number of regions.

\section{Cellular Automata Updating Rule}

Analyzed CA updating rule is based on the following observations:

- Pixels with greyscale values which are close to each other normally belong to the same region; pixels with greyscale values which are further apart normally belong to different regions;

- The state of a cell is influenced by the states of neighbour cells;

- A neighbour cell will give its state to the current cell only if

$\circ \quad$ the difference between their state values is less than a threshold $\epsilon$, and

- the difference in state value between the current cell and its neighbour cell with the furthest value (e.g. greyscale) is higher than a given threshold $\delta$.

Thus, the transition rule that we propose is conditioned not only by the geometry of the CA and the size and shape of each neighbourhood, but also by the state space.

In the transcription of the proposed $\mathrm{CA}$ rule, we use the following notations:

$M \quad$ current cell which will be updated by the CA rule according to the values of its neighbouring cells;

$A_{1} \quad$ neighbour of $M$ of closest greyscale value, with value less than the greyscale of $M$;

$A_{2} \quad$ neighbour of $M$ of closest greyscale value, with value greater than the greyscale of $M$;

$D_{1} \quad$ neighbour of $M$ of furthest greyscale value, with value less than the greyscale of $M$;

$D_{2} \quad$ neighbour of $M$ of furthest greyscale value, with value greater than the greyscale of $M$.

Taking into account the possible values ${ }^{1}$ of these cells, the current cell can have all these special neighbours or only some of them. The possible situations are:

- $\quad$ Both $D_{1}$ and $A_{1}$ exist, but there are no $A_{2}$ and $D_{2}$ : $D_{1}-A_{1}-M$

$$
\begin{aligned}
& M-A_{1}<\epsilon \\
& A_{1}-D_{1}>\delta
\end{aligned} \quad \Longrightarrow M=A_{1}
$$

- $\quad$ Both $D_{2}$ and $A_{2}$ exist, but there are no $A_{1}$ and $D_{1}$ : $M-A_{2}-D_{2}$

$$
\begin{aligned}
& A_{2}-M<\epsilon \\
& D_{2}-A_{2}>\delta
\end{aligned} \quad \Longrightarrow M=A_{2}
$$

\footnotetext{
${ }^{1}$ the value of a cell can represent a feature of the pixels - e.g. the gray-level, the RGB value, the gradient etc. - and the difference between two cells can be viewed in terms of distance between the considered feature
}

- $\quad$ All $A_{1}, A_{2}, D_{1}, D_{2}$ exist:

$$
\begin{array}{cc}
D_{1}-A_{1}-M-A_{2}-D_{2} & \\
M-A_{1}<\epsilon & \\
A_{1}-D_{1}>\delta & \Longrightarrow M=A_{1} \\
A_{2}-M<\epsilon & \\
D_{2}-A_{1}>\delta & \Longrightarrow M=A_{2} \\
M-A_{1}<\epsilon & \Longrightarrow M=A_{1} \\
A_{1}-D_{1}>\delta & \\
A_{2}-M<\epsilon & \\
D_{2}-A_{2}>\delta & \Longrightarrow M=A_{2}
\end{array}
$$

This rule causes each pixel to evolve in a direction away from the neighbour pixels with very different features (which could mean that they belong to a different region). Its application relies inherently on the size of each neighbourhood. The resulting automaton is able strongly to outline regions in an image, regardless of their dimension or shape.

Previous results [20]-[27] have shown how novel topologies and neighbourhoods can trigger good performance in CA tasks and they can be adapted so as to improve segmentation techniques. The four neighbourhood schemes that are being studied in this paper are formalized below and depicted in Figure 1. by:

The von Neumann neighbourhood of a cell $\left(x_{0}, y_{0}\right)$ is given

$N^{V}\left(x_{0}, y_{0}\right)=\left\{(x, y):\left|x-x_{0}\right|+\left|y-y_{0}\right| \leq r\right\}$.

The Moore neighbourhood of a cell $\left(x_{0}, y_{0}\right)$ is given by:

$N^{M}\left(x_{0}, y_{0}\right)=\left\{(x, y):\left|x-x_{0}\right| \leq r,\left|y-y_{0}\right| \leq r\right\}$.

In both cases, $r$ represents the range of the neighbourhood and $r=1$ for the standard case. In our analysis we investigate von Neumann and Moore neighbourhoods of range 1 and 2.

\section{NUMERICAL EXPERIMENTS}

We have carried out experiments using some real-world images. The Berkeley Segmentation Dataset [28] contains natural images with corresponding human segmentations (ground-truth). We have used a dozen of these images in order to validate our approach.

This work also uses a number of ten 3D volumes proposed for the image segmentation challenge of HVSMR 2016: MICCAI Workshop on Whole-Heart and Great Vessel Segmentation from 3D Cardiovascular MRI in Congenital Heart Disease [29] that will be hosted as part of the 19th conference on Medical Image Computing and Computer Assisted Intervention (MICCAI 2016).

Specifically, the data set [30] consists of pediatric cardiovascular magnetic resonance (CMR) images. The cases were acquired during clinical practice at the Boston Childrens Hospital, Boston, MA, USA and include various congenital heart defects. Cases where the patients have undergone interventions are also present. 


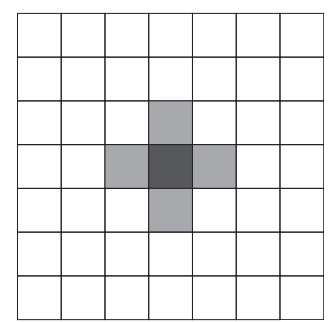

(a) von Neumann neighbourhood

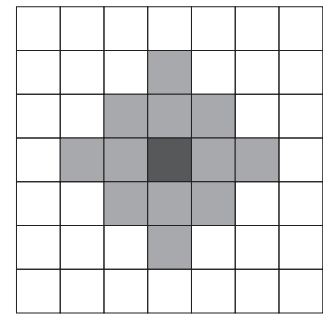

(c) extended von Neumann neighbourhood

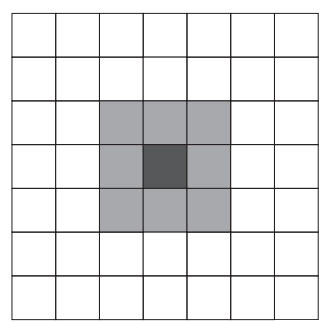

(b) Moore neighbourhood

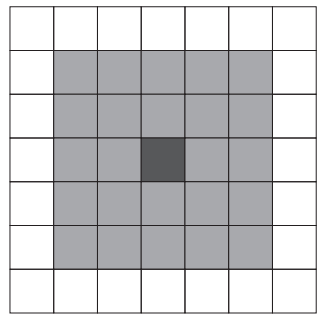

(d) extended Moore neighbourhood

Fig. 1: Different neighbourhood schemes for 2D Cellular Automata

The data set contains whole-heart images that include the myocardium, the blood pool and, in some cases, segments of other anatomical structures. The blood pool class includes the left and right atria, the left and right ventricles, the aorta, the pulmonary veins, the pulmonary arteries, and the superior and inferior vena cava. Vessels (except the aorta) are extended only a few centimeters past their origin. The myocardium class includes the thick muscle surrounding the two ventricles and the septum between them. Coronaries are not included in the blood pool class, and are labeled as myocardium if they travel within the ventricular myocardium. Moreover, a ground truth segmentation is also provided for each image. The ground truth images were obtained by performing manual segmentation in an approximate short-axis view. Even though all of the three planes were considered, the deciding factor was the quality of the segmentation in the short-axis view. The results of the segmentation, which was performed by a trained rater, was validated by two clinical experts.

Our experiments have been carried out using a twodimensional CA. The CA (width and height) dimensions correspond to the size of the image to be segmented. The process relies on a static CA (the set of cells and their interconnection pattern do not change with time) based on a grid of cells. Each cell of the CA can have one of 256 possible states which correspond to greyscale values. Even if the initial configuration (construction or random generation) often conditions the evolution of a CA, in our case the cells are initialized by the greyscale value of each pixel.

The conventional limit conditions for the $\mathrm{CA}$ can be periodic, reflective or with fixed value. We have chosen to work with fixed value borders. No boundary conditions are imposed for the evolution of the CA. Border cells simply have fewer neighbours than other cells.

\section{A. Performance measures}

It is very important to establish the way we define similar regions or segmentations. The obtained segmentations and their boundaries could be compact, discontinuous, smooth etc.

One of the metrics that we are using in our experiments is the Dice coefficient, which computes the overlap between regions, quantifying the similarity of two segmentations. This measure is especially useful when the volume changes are of great importance in the analysis process. The Dice similarity coefficient [31] (denoted as DICE in this paper) is computed as the ratio between the number of pixels belonging to the intersection (of two possible segmentations) and the average of their sizes.

Another frequently used measure for evaluating the segmentation performance is the Global Consistency Error (GCE) [28]. An error-based measure is actually an "opponent" to a similarity measure (two segmentations are identical if an errorbased measure is 0 ). This measure is computed as an average over the error of pixels/voxels belonging to two segmentations.

Some metrics that compute the distance between two segmented regions by taking into account the pixel location could be also considered. They quantify the dissimilarity of two segmentations and they are useful when the contours (the shapes of the boundary of the structure) are of importance for the image analysis. A distance value of 0 corresponds to a perfect match between the computed segmentation and the ground truth, while greater values indicate higher errors. In our approach, we consider the directed Hausdorff distance between two segmentations (called HDRFDST and defined as the maximal distance from a point in the first segmentation to a nearest point in the other one [32]) and the Mahalanobis Distance (MAHLNBS) [33] (that regards the correlation among all the pixels from the set that the considered two pixels are belonging).

A similarity measure able to evaluate both clusterings and 
classifications (because it is not based on labels) is the Rand Index (RNDIND), proposed in [34].

The similarity of two segmentations can be computed by taking into account their areas or volumes, also. In order to determine such a measure, a distance between two volumes must be defined (the similarity being 1 - this distance). A possible definition [35] for the volumetric distance is the ratio of the absolute volume difference and the sum of the compared volumes. Such a measure is useful when the segmentation purpose is to identify the changes in size since it is sensitive to miss-estimations of the segmented volume more than anything else. In the case of a single measurement, the volume error (two times the volume difference over the volume sum) can be used, while in the case of multiple measurements (when an average result is of interest) the absolute volume error can be used.

\section{B. Numerical results}

Figure 2 analyses in detail an image from the Berkeley database: original image, ground-truth and image segmented by a CA with the original rule after only 10 iterations. The visual analysis of the obtained segmented image shows the potential of the proposed rule.

In our experiments we aim to investigate how the parameters of the rule influence the performance of segmentation. On this purpose, various values have been searched for both parameters $(\epsilon$ and $\delta$ ) by using a random grid search. Grid search is simple to implement and parallelization is trivial. Furthermore, grid search is reliable in low dimensional spaces [36].

The two parameters being searched for, and the sets from which they are sampled, are given below:

- $\epsilon:\{5,6,7, \ldots, 20\}$

- $\delta:\{5,6,7, \ldots, 20\}$

When considering different neighbourhood schemes for the Berkely images, in most of the cases the best results are obtained when using the von Neumann neighbourhood, which is somehow unexpected because one would believe that receiving more information from the neighbouring cells would accelerate and improve the search process. Obtained results show that there is no reason for using more information than a simple von Neumann neighbourhood when updating the state of the cells. The obtained results for one such image are depicted in Figure 3.

Considering the von Neumann neighbourhood, we continue our study by applying the rule with different values of the parameters for several images from Berkely database. It can be observed in Figure 4 that there is no parameters pair that improves the Dice similarity for all images in the same way, rather, there is a set of different optimal parameters values for each shown image. These results are fairly intuitive and expected, as the gray values and value differences of the pixels in the considered images are different. These parameters should probably be computed based on the gray levels of the image, and this direction will be investigated as future work.

Regarding the medical images, out of the three data set options provided by the challenge, we have chosen to use the one consisting of cropped images that have been transformed into an approximate short-axis view. This data set contains gray-scale images with a 16 bits per pixel representation and 65,536 gray levels. The image dimensions vary among cases, with average dimensions of approximately 130 × 190 × 160 . The average size of an image is of about 33MB. Figure 5 provides an example of images existent in the data set. We provide the middle slice for each of the three axis.

The ground truth images contain three types of pixels: black pixels for the background, white pixels for the blood pool segmentation and gray pixels for the segmentation of the ventricular myocardium. Since, as a first approach, we only perform the segmentation of the blood pool, the gray pixels have been changed to black. The resulting ground truth for the images from Figure 5 can be seen in Figure 6.

We continue our study by investigating the results obtained for different values of the parameters combined with different types of neighbourhoods for the medical images. Obtained results are presented in Figure 7. First, the best Dice similarity is obtained when using the extended neighbourhoods. Moreover, extended Moore increases the similarity with the ground truth more than von Neumann neighbourhood. More information from the neighbourhood used to update a cell seems to improve the search process in this case.

Regarding the pairs of parameters which generate the best results, the conclusion drawn above can be extended here: there are no pairs of parameters that improves the similarity in the same way for all considered neighbourhoods.

The optimal parameters have been recorded for each neighbourhood and used in order to segment the image presented in Figure 5. Obtained segmented images for each considered neighbourhood are depicted in Figure 8.

In Table I we present the mean (over all images) and the corresponding confidence intervals of the results for all considered evaluation measures obtained in this experiment. The extended Moore neighbourhood and von Neumann neighbourhood compete for the best obtained results - half of the considered evaluation measures are best when using extended Moore neighbourhood, half of them are best when using von Neumann neighbourhood.

\section{CONCLUSIONS AND FURTHER WORK}

A multi-state CA-based image segmentation algorithm has been investigated by considering an original parameterized update rule and different neighbourhood topologies (von Neumann and Moore). Several real-world images have been used for conducting the study. The segmentation performance has been studied by using several supervised measures. In our study we investigate different values for the parameters involved within the update rule, different neighbourhood topologies for updating the cells, different evaluation measures and different real world images and we try to identify a correlation between them.

This study will continue with an analysis of the correlation between the gray levels (or other features) of the image and the values of the parameters on one hand, and the neighbourhood topology on the other hand. Other unsupervised performance measures will be investigated and analysed. 


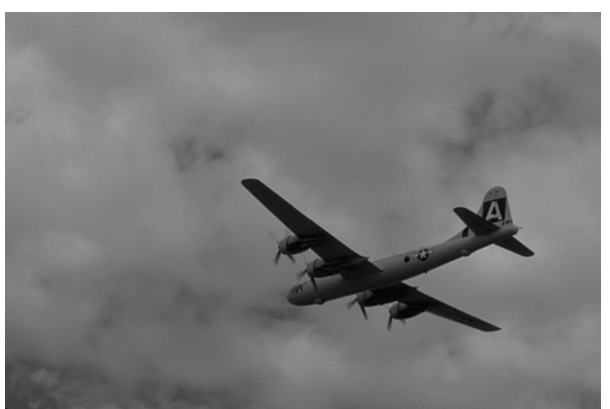

(a) original image

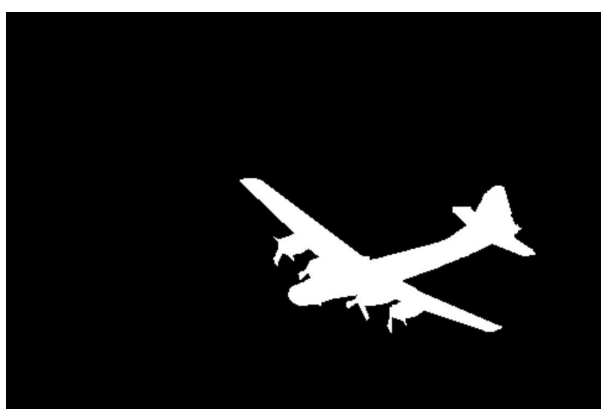

(b) ground truth image

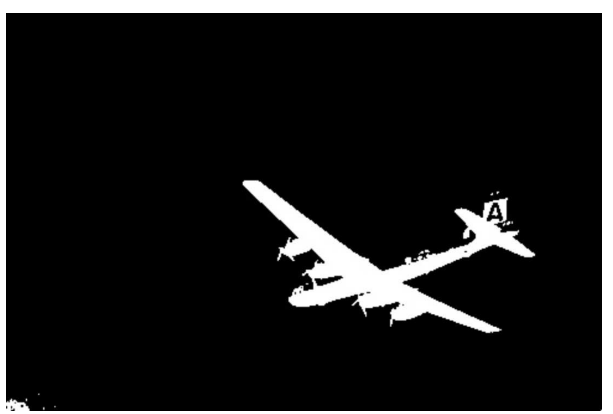

(c) image segmented by the proposed rule

Fig. 2: Image 3096 from Berkeley database

\begin{tabular}{c|cccccc} 
& Dice & GCE & HDR & Mahl & RndIndex & VolSim \\
\hline von Neumann & $0.778 \pm 0.099$ & $0.159 \pm 0.043$ & $35.612 \pm 4.629$ & $0.251 \pm 0.104$ & $0.831 \pm 0.052$ & $0.8603 \pm 0.1034$ \\
Moore & $0.780 \pm 0.098$ & $0.158 \pm 0.043$ & $34.542 \pm 4.418$ & $0.246 \pm 0.104$ & $0.832 \pm 0.052$ & $0.8621 \pm 0.1026$ \\
von Neumann Ext & $0.781 \pm 0.099$ & $0.156 \pm 0.044$ & $32.362 \pm 3.507$ & $0.245 \pm 0.104$ & $0.834 \pm 0.053$ & $0.8626 \pm 0.1039$ \\
Moore Ext & $0.783 \pm 0.099$ & $0.155 \pm 0.044$ & $32.702 \pm 4.317$ & $0.238 \pm 0.103$ & $0.836 \pm 0.053$ & $0.8631 \pm 0.1027$
\end{tabular}

TABLE I: Average performances over all tested medical 2D images for the considered neighbourhood topologies

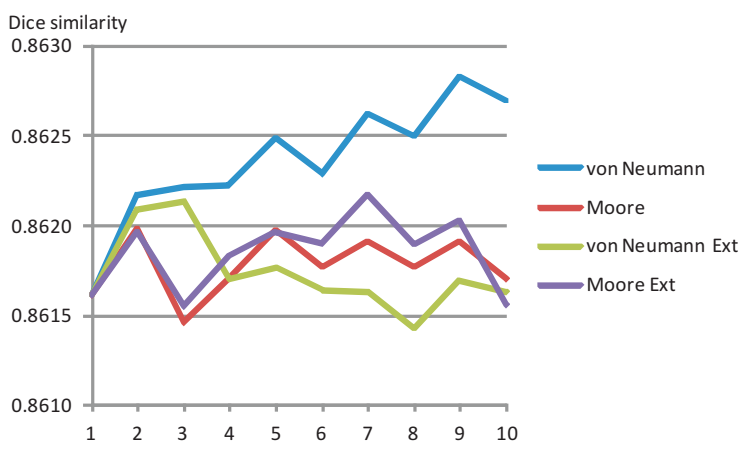

Fig. 3: Evolution of Dice similarity (image 3096) for the proposed rule — different neighbourhoods

\section{ACKNOWLEDGMENT}

This work was supported by a grant of the Romanian National Authority for Scientific Research and Innovation, CNCS - UEFISCDI, project number PN-II-RU-TE-2014-41130.

\section{REFERENCES}

[1] V. Vezhnevets and V. Konouchine, "Growcut" - interactive multi-label N-D image segmentation by cellular automata," 2005. [Online]. Available: http://citeseerx.ist.psu.edu/viewdoc/summary?doi=10.1.1.59.8092; http://graphics.cs.msu.su/en/publications/text/gc2005vk.pdf

[2] C. Kauffmann and N. Piche, "Seeded ND medical image segmentation by cellular automaton on GPU," Int. J. Computer Assisted Radiology and Surgery, vol. 5, no. 3, pp. 251-262, 2010. [Online]. Available: http://dx.doi.org/10.1007/s11548-009-0392-0

[3] S. Wongthanavasu, "Cellular automata for medical image processing," Apr. 112011.

[4] A. Hamamci, N. Kucuk, K. Karaman, K. Engin, and G. B. Ünal, "Tumor-cut: Segmentation of brain tumors on contrast enhanced MR images for radiosurgery applications," IEEE Trans. Med. Imaging, vol. 31, no. 3, pp. 790-804, 2012. [Online]. Available: http://dx.doi.org/10.1109/TMI.2011.2181857

[5] M. Sonka, V. Hlavac, and R. Boyle, Image, Processing, Analysis and Machine Vision, ser. Chapman \& Hall Computing Series. London, United Kingdom: Chapman \& Hall, 1993.

[6] K. S. Fu and J. K. Mui, "A survey on image segmentation," Pattern Recognition, vol. 13, pp. 3-16, 1981, earliest survey of segmentation, mainly thresholding.

[7] K. McGuinness and N. E. O'Connor, "A comparative evaluation of interactive segmentation algorithms," Pattern Recognition, vol. 43, no. 2, pp. 434-444,, Feb. 2010. [Online]. Available: http://dx.doi.org/10.1016/j.patcog.2009.03.008

[8] P. L. Rosin, "Training cellular automata for image processing," in SCIA, 2005, pp. 195-204. [Online]. Available: http://dx.doi.org/10.1007/11499145_22 


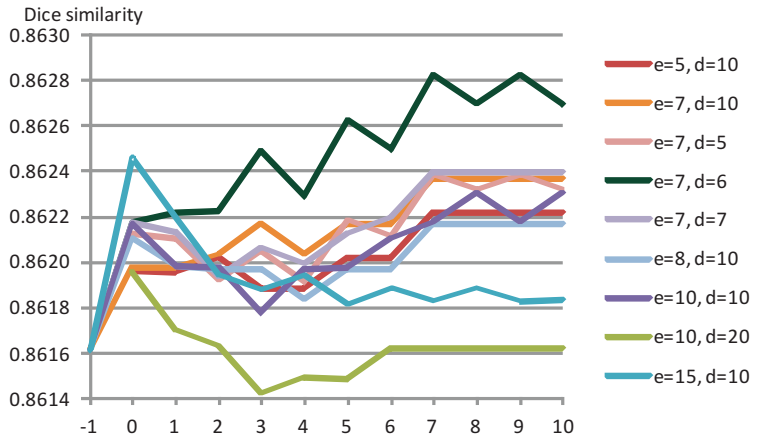

(a) image 3096

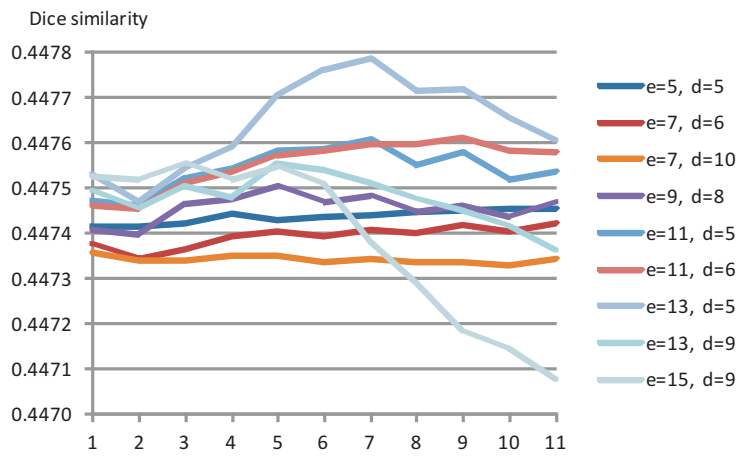

(c) image 100098

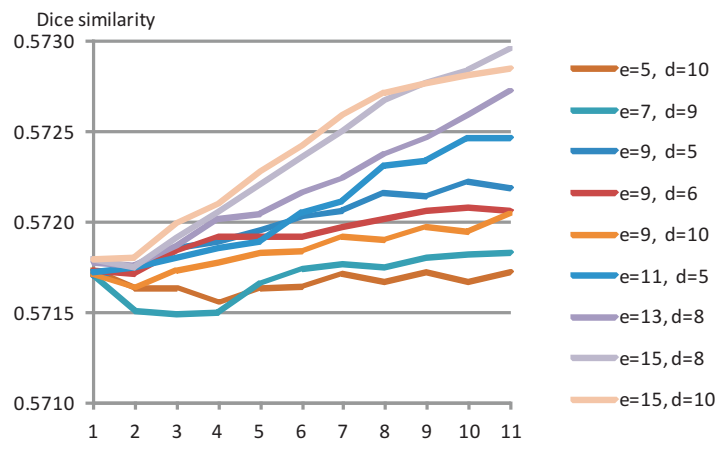

(b) image 3063

Fig. 4: Evolution of Dice similarity when using von Neumann neighbourhood
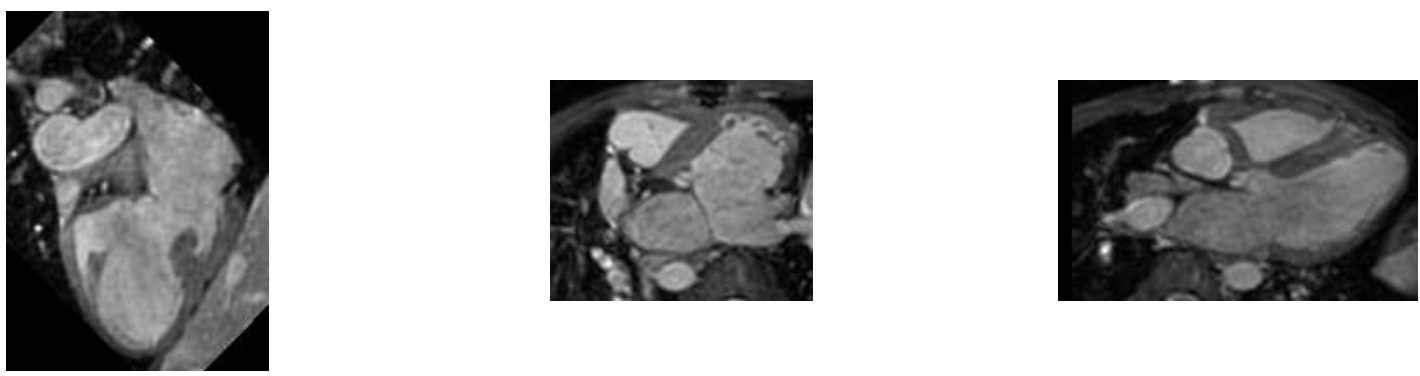

Fig. 5: Slices through sample images
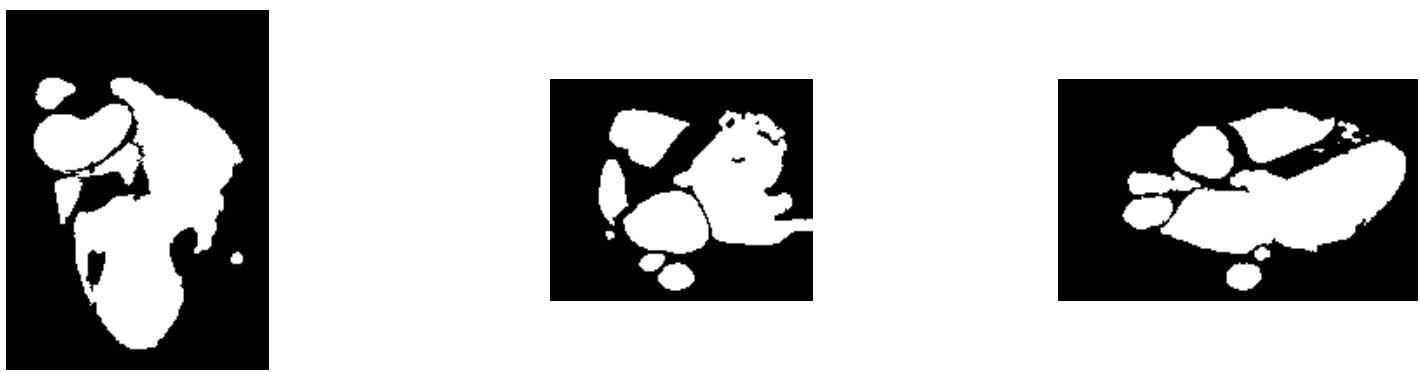

Fig. 6: Slices through sample GT images 


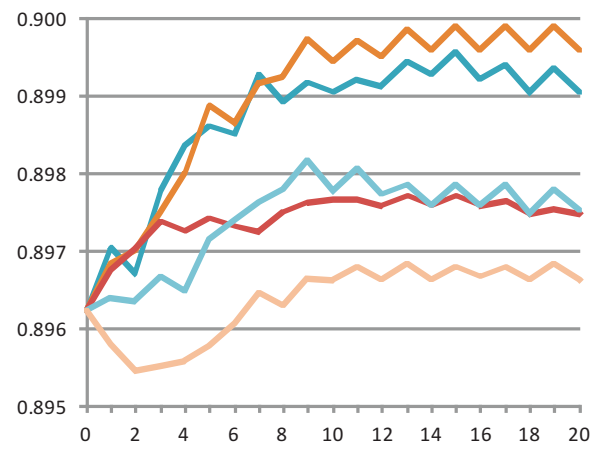

(a) von Neumann neighbourhood

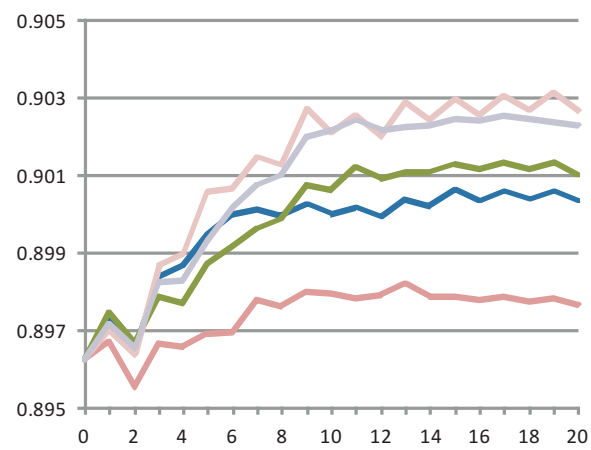

(c) Moore neighbourhood

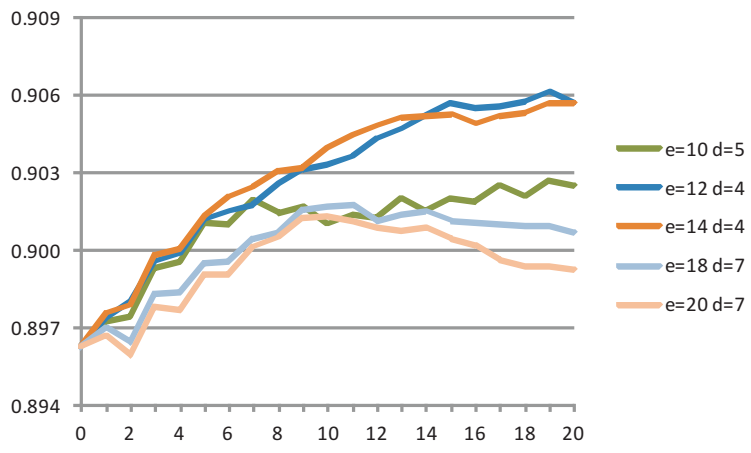

(b) von Neumann Ext neighbourhood

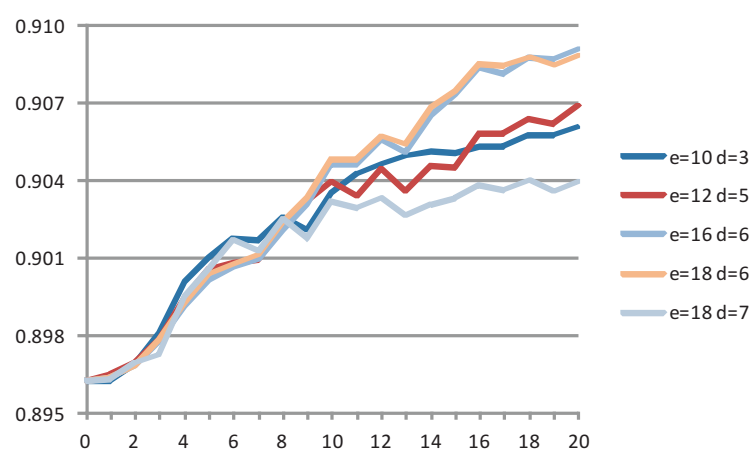

(d) Moore Ext neighbourhood

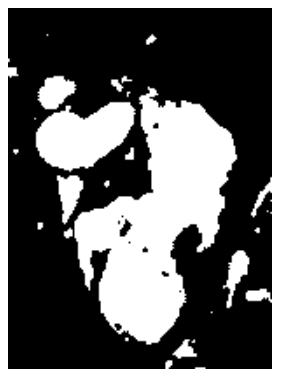

(a) von Neumann neighbourhood

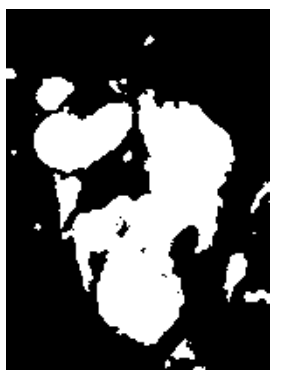

(c) Moore neighbourhood
Fig. 7: Evolution of Dice similarity
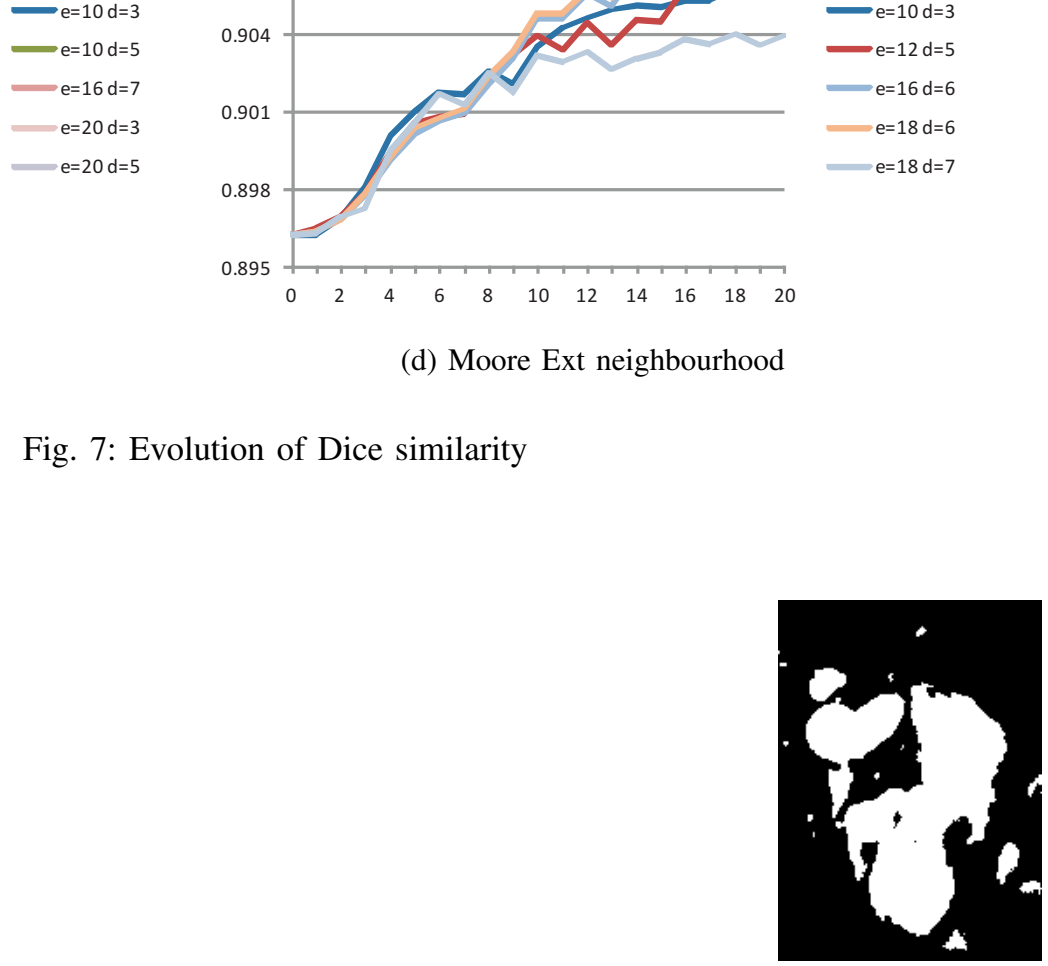

(b) von Neumann Ext neighbourhood

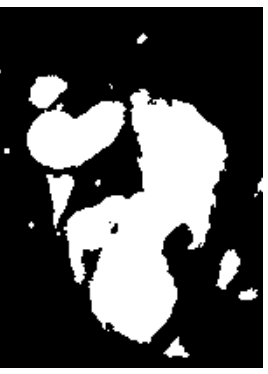

(d) Moore Ext neighbourhood

Fig. 8: Segmented image by CA 
[9] C. C. Christiyana, V. Rajamani, and A. U. Devi, "Article: Ultra sound kidney image retrieval using time efficient one dimensional glcm texture feature," IJCA Special Issue on Advanced Computing and Communication Technologies for HPC Applications, vol. ACCTHPCA, no. 4, pp. 12-17, July 2012, full text available.

[10] M. Diwakar, P. K. Patel, and K. Gupta, "Cellular automata based edge-detection for brain tumor," in ICACCI. IEEE, 2013, pp. 53-59. [Online]. Available: http://ieeexplore.ieee.org/xpl/mostRecentIssue.jsp?punumber=6621059

[11] D. R. Nayak, S. K. Sahu, and J. Mohammed, "A cellular automata based optimal edge detection technique using twenty-five neighborhood model," CoRR, vol. abs/1402.1348, 2014. [Online]. Available: http://arxiv.org/abs/1402.1348

[12] D. Safia, D. Oussama, and B. Chawki, "Image segmentation using continuous cellular automata," in Programming and Systems (ISPS), 2011 10th International Symposium on, April 2011, pp. 94-99.

[13] D. Safia and B. Chawki, "Image segmentation using an emergent complex system: Cellular automata," in Systems, Signal Processing and their Applications (WOSSPA), 2011 7th International Workshop on, May 2011, pp. 207-210.

[14] M. H. Mofrad, S. Sadeghi, A. Rezvanian, and M. R. Meybodi, "Cellular edge detection: Combining cellular automata and cellular learning automata," $\{A E U\}$ - International Journal of Electronics and Communications, vol. 69, no. 9, pp. 1282 - 1290, 2015. [Online]. Available: http://www.sciencedirect.com/science/article/pii/S1434841115001454

[15] P. L. Rosin, "Image processing using 3-state cellular automata," Computer Vision and Image Understanding, vol. 114, no. 7, pp. 790-802, 2010. [Online]. Available: http://dx.doi.org/10.1016/j.cviu.2010.02.005

[16] H.-O. Peitgen, H. Jürgens, and D. Saupe, Chaos and fractals - new frontiers of science. Springer, 1992.

[17] P. Bak, C. Tang, and K. Wiesenfeld, "Self-organized criticality: An explanation of 1/f noise," Phys. Rev. Lett., vol. 59, pp. 381-384, 1987.

[18] J. M. Greenberg and S. P. Hastings, "Spatial patterns for discrete models of diffusion in excitable media," SIAM Journal on Applied Mathematics, vol. 34, pp. 515-523, 1978.

[19] S. Wolfram, "Statistical mechanics of cellular automata," Rev. Mod. Phys., vol. 55, no. 3, pp. 601-644, 1983.

[20] D. J. Watts and S. H. Strogatz, "Collective dynamics of 'small-world' networks," Nature, vol. 393, pp. 440-442, 1998.

[21] D. J. Watts, Small Worlds. Princeton): (Princeton University Press, 1999.

[22] C. Darabos, M. Giacobini, and M. Tomassini, "Performance and robustness of cellular automata computation on irregular networks," Advances in Complex Systems, vol. 10, no. supp01, pp. 85-110, 2007. [Online]. Available: http://dx.doi.org/10.1142/S0219525907001124

[23] C. Darabos, M. Tomassini, F. D. Cunto, P. Provero, J. H. Moore, and M. Giacobini, "Toward robust network based complex systems: from evolutionary cellular automata to biological models," Intelligenza Artificiale, vol. 5, no. 1, pp. 37-47, 2011. [Online]. Available: http://dx.doi.org/10.3233/IA-2011-0003
[24] M. Tomassini, M. Giacobini, and C. Darabos, "Evolution and dynamics of small-world Cellular Automata," Complex Systems, vol. 1, no. 15, pp. 261-284, 2005.

[25] A. Gog and C. Chira, "Dynamics of networks evolved for cellular automata computation," in HAIS (2), ser. Lecture Notes in Computer Science, E. Corchado, V. Snásel, A. Abraham, M. Wozniak, M. Graña, and S.-B. Cho, Eds., vol. 7209. Springer, 2012, pp. 359-368. [Online]. Available: http://dx.doi.org/10.1007/978-3-642-28931-6

[26] A. Andreica and C. Chira, "Evolution and dynamics of node-weighted networks for cellular automata computation," Logic Journal of the $I G P L$, vol. 23, no. 3, pp. 400-409, 2015. [Online]. Available: http://dx.doi.org/10.1093/jigpal/jzv005

[27] _ _ "Using a hybrid cellular automata topology and neighborhood in rule discovery," in HAIS, ser. Lecture Notes in Computer Science, J.-S. Pan, M. M. Polycarpou, M. Wozniak, A. C. P. L. F. Carvalho, H. Quintián-Pardo, and E. Corchado, Eds., vol. 8073. Springer, 2013, pp. 669-678. [Online]. Available: http://dx.doi.org/10.1007/978-3-64240846-5

[28] D. R. Martin, C. C. Fowlkes, D. Tal, and J. Malik, "A database of human segmented natural images and its application to evaluating segmentation algorithms and measuring ecological statistics," in ICCV, 2001, pp. II: 416-423. [Online]. Available: http://dx.doi.org/10.1109/ICCV.2001.937655

[29] H. 2016, "Miccai workshop on whole-heart and great vessel segmentation from 3d cardiovascular mri in congenital heart disease," 2016. [Online]. Available: http://segchd.csail.mit.edu/index.html

[30] D. F. Pace, A. V. Dalca, T. Geva, A. J. Powell, M. H. Moghari, and P. Golland, "Interactive whole-heart segmentation in congenital heart disease," in MICCAI (3), ser. Lecture Notes in Computer Science, N. Navab, J. Hornegger, W. M. W. III, and A. F. Frangi, Eds., vol. 9351. Springer, 2015, pp. 80-88. [Online]. Available: http://dx.doi.org/10.1007/978-3-319-24574-4

[31] L. R. Dice, "Measures of the amount of ecologic association between species," Ecology, vol. 26, no. 3, pp. 297-302, 1945. [Online]. Available: http://www.jstor.org/stable/1932409

[32] F. Hausdorff, Grundzüge der Mengenlehre. New York: Chelsea, 1914, reprinted by Chelsea, 1949.

[33] P. C. Mahalanobis, "On the generalised distance in statistics," in Proc. of the Nat. Inst. of Sci. of India, 1936, pp. 49-55, published as Proc. of the Nat. Inst. of Sci. of India, volume 2, number 1.

[34] W. M. Rand, "Objective criteria for the evaluation of clustering methods," American Statistical Association Journal, vol. 66, no. 336, pp 846-850, 1971.

[35] A. A. Taha and A. Hanbury, "Metrics for evaluating 3D medical image segmentation: analysis, selection, and tool," $B M C$ Medical Imaging, vol. 15, p. 29, 2015. [Online]. Available: http://dx.doi.org/10.1186/s12880-015-0068-x

[36] J. Bergstra and Y. Bengio, "Random search for hyper-parameter optimization," Journal of Machine Learning Research, vol. 13, pp. 281-305, 2012. [Online]. Available: http://dl.acm.org/citation.cfm?id=2188395 